TITLE:

\title{
Dynamics and rheology of a supercooled polymer melt in shear flow
}

$\operatorname{AUTHOR}(S)$ :

Yamamoto, R; Onuki, A

\section{CITATION:}

Yamamoto, R ... [et al]. Dynamics and rheology of a supercooled polymer melt in shear flow. JOURNAL OF CHEMICAL PHYSICS 2002, 117(5): 2359-2367

\section{ISSUE DATE:}

2002-08-01

URL:

http://hdl.handle.net/2433/50115

\section{RIGHT:}

Copyright 2002 American Institute of Physics. This article may be downloaded for personal use only. Any other use requires prior permission of the author and the American Institute of Physics. 


\title{
Dynamics and rheology of a supercooled polymer melt in shear flow
}

\author{
Ryoichi Yamamoto a) and Akira Onuki \\ Department of Physics, Kyoto University, Kyoto 606-8502, Japan
}

(Received 15 March 2002; accepted 2 May 2002)

\begin{abstract}
Using molecular dynamics simulations, we study dynamics of a model polymer melt composed of short chains with bead number $N=10$ in supercooled states. In quiescent conditions, the stress relaxation function $G(t)$ is calculated, which exhibits a stretched exponential relaxation on the time scale of the $\alpha$ relaxation time $\tau_{\alpha}$ and ultimately follows the Rouse dynamics characterized by the time $\tau_{R} \sim N^{2} \tau_{\alpha}$. After application of shear $\dot{\gamma}$, transient stress growth $\sigma_{x y}(t) / \dot{\gamma}$ first obeys the linear growth $\int_{0}^{t} d t^{\prime} G\left(t^{\prime}\right)$ for strain less than 0.1 but saturates into a non-Newtonian viscosity for larger strain. In steady states, shear thinning and elongation of chains into ellipsoidal shapes take place for shear $\dot{\gamma}$ larger than $\tau_{R}^{-1}$. In such strong shear, we find that the chains undergo random tumbling motion taking stretched and compact shapes alternatively. We examine the validity of the stressoptical relation between the anisotropic parts of the stress tensor and the dielectric tensor, which are violated in transient states due to the presence of a large glassy component of the stress. We furthermore introduce time-correlation functions in shear to calculate the shear-dependent relaxation times, $\tau_{\alpha}(T, \dot{\gamma})$ and $\tau_{R}(T, \dot{\gamma})$, which decrease nonlinearly as functions of $\dot{\gamma}$ in the shear-thinning regime. (C) 2002 American Institute of Physics. [DOI: 10.1063/1.1488589]
\end{abstract}

\section{INTRODUCTION}

The dynamics and rheology of glassy polymers are known to be very complicated and are still not well understood. We summarize salient features of such systems below.

First, in the linear response regime, thermal relaxations of the chain conformations occur from microscopic to macroscopic time scales, as revealed in measurements of stress and dielectric responses. ${ }^{1,2}$ In a relatively early stage, the stress relaxation function $G(t)$, which describes linear response to small shear deformations, can be fitted to the Kohlrausch-Williams-Watts (KWW) form:

$$
G_{G}(t)=G_{0} \exp \left[-\left(t / \tau_{s}\right)^{c}\right],
$$

after a microscopic transient time $t_{\text {tra }}$. The time $\tau_{s}\left(\gg \tau_{\text {tra }}\right)$ is of the order of the structural $\alpha$ relaxation time $\tau_{\alpha}$ [to be defined in Eq. (3.8)], which grows dramatically as the temperature $T$ is lowered toward the glass transition temperature $T_{g}$. When the time $t$ considerably exceeds $\tau_{s}$, the relaxation of the chain conformations is relevant and is well described by the Rouse or reptation dynamics, depending on whether $N<N_{e}$ or $N>N_{e}$, respectively. ${ }^{3}$ Here $N$ is the polymerization index and $N_{e}$ is that between entanglements on a chain. For short chain systems with $N<N_{e}$, the overall behavior in the time region $t \gg t_{\text {tra }}$ may be expressed as

$$
G(t)=G_{G}(t)+G_{R}(t) .
$$

The $G_{R}(t)$ is the stress time-correlation function in the Rouse model whose terminal relaxation time $\tau_{R}$ is of order $N^{2} \tau_{\alpha}$. For entangled chain systems with $N>N_{e}$, the KWW function in Eq. (1.1) is followed by the power-law decay:

$$
G(t) \cong e^{-1} G_{0}\left(t / \tau_{s}\right)^{-\nu}
$$

${ }^{a)}$ Electronic mail: ryoichi@scphys.kyoto-u.ac.jp with $\quad \nu \sim 0.5$ until the rubbery plateau $G(t) \simeq G_{N}^{(0)}$ is reached, ${ }^{1,2}$ where $G_{N}^{(0)}$ assumes the modulus $n k_{B} T / N_{e}$ of entangled polymers with $n$ being the bead number density. Ultimately, $G(t)$ follows the reptation relaxation $G_{\text {rep }}(t)$ on the time scale of a very long reptation time $\tau_{\text {rep }}$. These hierarchical relaxations arise from rearrangements of jammed atomic configurations and subsequent evolution of chain conformations. They also give rise to the corresponding characteristic behaviors in the frequency-dependent shear modulus $G^{*}(\omega) \equiv i \omega \int_{0}^{\infty} d t e^{-i \omega t} G(t)$, depending on the frequency $\omega$ relative to the inverse characteristic times introduced. ${ }^{1-3}$

Second, in the nonlinear response regime, glassy fluids generally exhibit highly viscous non-Newtonian flow close to (but above) $T_{g}$ even if they are low-molecular-weight fluids. ${ }^{4}$ In such fluids, if $\dot{\gamma}>\tau_{\alpha}^{-1}$, atomic rearrangements are induced not by thermal agitations but by externally applied shear. ${ }^{5-7}$ In chain systems without entanglements, on one hand, shear thinning occurs at sufficiently high (but sometimes unrealistically large) shear rates due to chain elongation. ${ }^{8-12}$ In entangled polymers, on the other hand, shear thinning occurs at a very small shear larger than $\tau_{\text {rep }}^{-1}$, where disentanglements are induced by shear. ${ }^{3}$ Thus, supercooled chain systems are most easily driven into a nonlinear response regime even by extremely small shear, though the crossover shear stress from linear to nonlinear regimes may not be very small. Furthermore, in glassy fluids below $T_{g}$, plastic deformations are often induced in the form of largescale shear bands above a yield stress (corresponding to a few $\%$ strain). ${ }^{13,14}$ It is of great importance to understand how these nonlinear effects occur depending on $\dot{\gamma}, T$, and $N$.

Third, in rheological experiments on polymers, use has been made of the stress-optical relation between the deviatoric (anisotropic) parts of the dielectric tensor $\epsilon_{\alpha \beta}$ (at optical frequencies) and the average stress tensor $\sigma_{\alpha \beta} \cdot{ }^{3,15} \mathrm{In}$ 
shear flow with mean velocity $\dot{\gamma} y$ in the $x$ direction, it is expressed as

$$
\epsilon_{x y}=C_{0} \sigma_{x y}, \quad \epsilon_{\alpha \alpha}-\epsilon_{\beta \beta}=C_{0}\left(\sigma_{\alpha \alpha}-\sigma_{\beta \beta}\right),
$$

where $C_{0}$ is called the stress-optical coefficient. For melts this relation excellently holds at relatively high $T\left(>T_{g}\right.$ ) for general time-dependent nonlinear shear deformations. If measurements are made in steady states, it holds even close to $T_{g} \cdot{ }^{16}$ For its validity, we need to require that the form contribution to $\epsilon_{\alpha \beta}$ is negligible as compared to the intrinsic contribution $^{15}$ and that the glassy part of the stress is negligible as compared to the usual entropic part. Thus, it is violated when the form part is relevant such as in polymer solutions close to the demixing critical point or when measurements are made in transient states close to $T_{g}$. In the latter case, as is evident from the enhancement of $\tau_{s}$ in Eq. (1.1), the glassy part of the stress is dominant for relatively rapid deformations. ${ }^{16-19}$

While the predictive power of analytic theories in polymer science is still poor, computer simulations ${ }^{20-22}$ can provide us with a useful tool to investigate the microscopic origins of experimentally observed macroscopic phenomena. In quiescent states, diffusive motions in supercooled melts have been extensively studied using molecular dynamics $(\mathrm{MD})^{23-27}$ and Monte Carlo ${ }^{28-30}$ simulations. In another application, nonequilibrium molecular dynamics (NEMD) simulations have been useful to investigate chain deformations and rheology in flow. ${ }^{8-12,17,31}$ In particular, Kröger et al. ${ }^{17}$ studied the molecular mechanisms of the violations of the stress-optical behavior for a melt consisting of $M$ $=260$ chains with bead number $N=30$ after application of elongational flow.

In this article, we will present results of very long MD simulations to study linear and nonlinear dynamics of a supercooled polymer melt in the absence and presence of shear flow. Long simulation times are needed to calculate the terminal relaxation of $G(t)$, which has not yet been undertaken in the literature. As a new finding, we will show that each chain in our melt system is changing its orientation (tumbling) randomly in shear flow. Use will be made of techniques and concepts introduced in our previous papers on supercooled binary mixtures under shear flow. ${ }^{5-7}$ Some of our results were published elsewhere. ${ }^{7,32}$

\section{MODEL AND SIMULATION METHOD}

Our system is composed of $M=100$ chains with $N$ $=10$ beads confined in a cubic box with length $L=10 \sigma$ and volume $V=L^{3}=10^{3} \sigma^{3}$. The number density is fixed at $n$ $=N M / V=1 / \sigma^{3}$, which results in severely jammed configurations at low $T$. All the bead particles interact with a truncated Lennard-Jones potential defined by ${ }^{20}$

$$
U_{\mathrm{LJ}}(r)=4 \epsilon\left[\left(\frac{\sigma}{r}\right)^{12}-\left(\frac{\sigma}{r}\right)^{6}\right]+\epsilon \quad\left(r<2^{1 / 6} \sigma\right) .
$$

The right-hand side is minimum at $r=2^{1 / 6} \sigma$ and the potential is truncated for larger $r\left[U_{\mathrm{LJ}}(r)=0\right.$ for $\left.r>2{ }^{1 / 6} \sigma\right]$. By using the repulsive part of the Lennard-Jones potential only, we may prevent spatial overlap of the particles. ${ }^{20}$ Consecutive beads on each chain are connected by an anharmonic spring of the form:

$$
U_{F}(r)=-\frac{1}{2} k_{c} R_{0}^{2} \ln \left[1-\left(r / R_{0}\right)^{2}\right]
$$

with $k_{c}=30 \epsilon / \sigma^{2}$ and $R_{0}=1.5 \sigma$. In our simulation the bond lengths $b_{j}^{k} \equiv\left|\boldsymbol{R}_{j}^{k}-\boldsymbol{R}_{j+1}^{k}\right|(1 \leqslant j \leqslant N-1)$ between consecutive beads on the same chain $k$ were very close to the minimum distance $b_{\min } \cong 0.96 \sigma$ of the sum $U_{\mathrm{LJ}}(r)+U_{F}(r)$. The deviations $b_{j}^{k}-b_{\text {min }}$ were only on the order of a few $\%$ of $b_{\min }$ for any $T$ and $\dot{\gamma}$ realized in our study.

Microscopic expressions for physical quantities can be expressed in terms of the momentum and position vectors of the $j$ th bead on the $k$ th chain, $\boldsymbol{R}_{j}^{k}$ and $\boldsymbol{p}_{j}^{k}$, where $j$ $=1, \ldots, N$ and $k=1, \ldots, M$. For example, the space integral of the microscopic stress tensor reads

$$
\begin{aligned}
\Pi_{\alpha \beta}^{\mathrm{T}}(t)= & \frac{1}{m} \sum_{k=1}^{M} \sum_{j=1}^{N} p_{j \alpha}^{k} p_{j \beta}^{k}-\sum_{\text {all pairs }} U_{\mathrm{LJ}}^{\prime}(\xi) \frac{\xi_{\alpha} \xi_{\beta}}{\xi} \\
& -\sum_{k=1}^{M} \sum_{j=1}^{N-1} U_{F}^{\prime}(\xi) \frac{\xi_{\alpha} \xi_{\beta}}{\xi}
\end{aligned}
$$

where $m$ is the mass of a bead, $U_{\mathrm{LJ}}^{\prime}(\xi)=d U_{\mathrm{LJ}}(\xi) / d \xi$, and $U_{F}^{\prime}(\xi)=d U_{F}(\xi) / d \xi$. Here $\boldsymbol{\xi}=\left(\xi_{x}, \xi_{y}, \xi_{z}\right)$ in the right-hand side represents the relative vector $\boldsymbol{R}_{j}^{k}-\boldsymbol{R}_{j^{\prime}}^{k^{\prime}}$ between the two beads, $\boldsymbol{R}_{j}^{k}$ and $\boldsymbol{R}_{j^{\prime}}^{k^{\prime}}$, in the second term and the relative vector $\boldsymbol{R}_{j}^{k}-\boldsymbol{R}_{j+1}^{k}$ between the two consecutive beads, $\boldsymbol{R}_{j}^{k}$ and $\boldsymbol{R}_{j+1}^{k}$, of the same chain in the third term. To avoid cumbersome notation, we will write the bead positions simply as $\boldsymbol{R}_{j}$ ( $j$ $=1, \ldots, N)$ suppressing the index $k$. When they will appear in the statistical averages $\langle\cdots\rangle$, the average over all the chains $\sum_{k=1}^{M}(\cdots) / M$ will be implied even if not written explicitly. Furthermore, it is convenient here to introduce the usual notation $\sigma_{\alpha \beta}$ for the stress tensor by

$$
\frac{1}{V} \Pi_{\alpha \beta}^{\mathrm{T}}=p \delta_{\alpha \beta}-\sigma_{\alpha \beta},
$$

where $p$ is the pressure and the second term is deviatoric. ${ }^{3}$ The $\sigma_{\alpha \beta}$ has already appeared in the stress-optical relation (1.4).

Hereafter we will measure space and time in units of $\sigma$ and $\tau_{0} \equiv\left(m \sigma^{2} / \epsilon\right)^{1 / 2}$. The temperature $T$ will be measured in units of $\epsilon / k_{B}$. The original units will also be used when confusion may occur. Our simulations cover normal ( $T$ $=1.0)$ and supercooled $(T=0.2)$ states with and without shear flow $\left(\dot{\gamma}=0,10^{-4}, 10^{-3}, 10^{-2}\right.$, and $\left.10^{-1}\right)$. Simulation data were taken after very long equilibration periods $\left(\sim 10^{2} \tau_{R} \simeq 5 \times 10^{6}\right.$ at $\left.T=0.2\right)$ so that no appreciable aging (slow equilibration) effect was detected in the course of taking data in various quantities such as the pressure or the density time-correlation functions. (i) In quiescent cases, we impose the microcanonical condition and integrate Newton's equations of motion:

$$
\frac{d}{d t} \boldsymbol{R}_{j}=\frac{1}{m} \boldsymbol{p}_{j}, \frac{d}{d t} \boldsymbol{p}_{j}=\boldsymbol{f}_{j},
$$


where $f_{j}$ is the force acting on the particle $j$ due to the potentials. Integration was performed with time increment $\Delta t$ $=0.005$ under the periodic boundary condition. Long time simulations of order $10^{2} \tau_{R}$, which corresponds to $10^{9} \mathrm{MD}$ steps for $T=0.2$, were performed. In the previous simulations, ${ }^{20,23-25,28-30}$ however, the integrated times did not much exceed $\tau_{R}$ in supercooled states. (ii) In the presence of shear, rewriting the momentum deviations $\boldsymbol{p}_{j}$ $-m \dot{\gamma} Y_{j} \boldsymbol{e}_{x}$ from the mean flow as $\boldsymbol{p}_{j}$, we integrated the socalled SLLOD equations of motion: ${ }^{33,34}$

$$
\begin{aligned}
& \frac{d}{d t} \boldsymbol{R}_{j}=\frac{1}{m} \boldsymbol{p}_{j}+\dot{\gamma} Y_{j} \boldsymbol{e}_{x}, \\
& \frac{d}{d t} \boldsymbol{p}_{j}=\boldsymbol{f}_{j}-\dot{\gamma} p_{y_{j}} \boldsymbol{e}_{x}-\hat{\zeta} \boldsymbol{p}_{j},
\end{aligned}
$$

where $\boldsymbol{e}_{x}$ is the unit vector in the $x$ (flow) direction, $\boldsymbol{R}_{j}$ $=\left(X_{j}, Y_{j}, Z_{j}\right)$, and $\boldsymbol{p}_{j}=\left(p_{x j}, p_{y j}, p_{z j}\right)$. The friction coefficient $\hat{\zeta}$ was set equal to

$$
\hat{\zeta}=\sum_{j}\left(\boldsymbol{f}_{j} \cdot \boldsymbol{p}_{j}-\dot{\gamma} p_{x j} p_{y j}\right) / \sum_{j} \boldsymbol{p}_{j}^{2} .
$$

The temperature $T\left(\equiv \Sigma_{j} \boldsymbol{p}_{j}^{2} / 3 m N M\right)$ could then be kept at a desired value. The time increment was $\Delta t=0.0025$. After an equilibration run in a quiescent state for $t<0$, we gave all the particles the average flow velocity $\dot{\gamma} Y_{j} \boldsymbol{e}_{x}$ at $t=0$ and then imposed the Lee-Edwards boundary condition ${ }^{33,34}$ to maintain the shear flow. Steady sheared states were realized after transient relaxations.

\section{DYNAMICS IN QUIESCENT STATES}

Although it is highly nontrivial, it has been confirmed by computer simulations ${ }^{20,23-25,28-30,35}$ that the single-chain near-equilibrium dynamics in unentangled melts can be reasonably well described by (or mapped onto) the simple Rouse model. In the Rouse dynamics, the relaxation time of the $p$ th mode of a chain is expressed in terms of a friction coefficient $\zeta$ and a segment length $b$ as ${ }^{36}$

$$
\tau_{p}=\zeta b^{2} /\left[12 k_{B} T \sin ^{2}(\pi p / 2 N)\right],
$$

where $1 \leqslant p \leqslant N-1$. The Rouse relaxation time $\tau_{R}$ is the slowest relaxation time:

$$
\tau_{R} \equiv \tau_{1} \cong N^{2} \zeta b^{2} /\left(3 \pi^{2} k_{B} T\right) .
$$

The segment length $b$ in the corresponding Rouse model may be related to the variance of the end-to-end vector of a chain $\boldsymbol{P} \equiv \boldsymbol{R}_{N}-\boldsymbol{R}_{1}$ in our microscopic model by

$$
\left\langle|\boldsymbol{P}|^{2}\right\rangle=b^{2}(N-1) .
$$

As a result, $b$ is dependent on $T$ but its dependence turns out to be weak as $b=1.17,1.18,1.19$ for $T=1.0,0.4,0.2$, respectively. Note that $b$ is larger than the minimum distance $b_{\min } \cong 0.96$ of the bond potential. Let us consider the timecorrelation function of $\boldsymbol{P}(t)$ :

$$
C(t)=\left\langle\boldsymbol{P}\left(t+t_{0}\right) \cdot \boldsymbol{P}\left(t_{0}\right)\right\rangle /\left\langle|\boldsymbol{P}|^{2}\right\rangle,
$$

which is normalized such that $C(0)=1$. Here $C(t)$ should be independent of the initial time $t_{0}$ in steady states in the limit of large system size. However, our system is not very large,

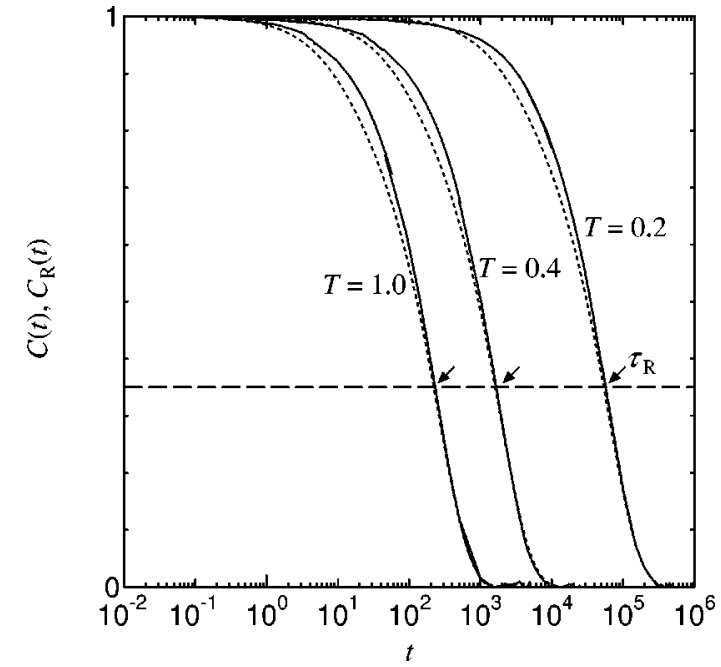

FIG. 1. Normalized end-to-end vector time-correlation function $C(t)$ in (3.4) for $T=1.0,0.4$, and 0.2 on a semi logarithmic scale. The dotted lines are the results of the Rouse model (3.5). The Rouse time $\tau_{R}$ in Eq. (3.2) is indicated by arrows.

so we took the average over the initial time $t_{0}$. This statistical averaging will not be mentioned hereafter in showing our MD results of time-correlation functions. In the Rouse dynamics $C(t)$ is calculated as

$$
C_{R}(t)=\frac{2}{(N-1) N} \sum_{\text {odd } p} \cot ^{2}\left(\frac{\pi p}{2 N}\right) e^{-t / \tau_{p}},
$$

where the summation is over odd $p$ but the first term ( $p$ $=1$ ) is dominant in the whole time region (so we may determine $\tau_{R}$ by $C\left(\tau_{R}\right)=e^{-1}$ ]. Figure 1 shows that our MD data of $C(t)$ can be fitted to $C_{R}(t)$. The $\tau_{R}$ thus determined increases drastically with lowering $T$ as $\tau_{R}=250,1800$, and $6 \times 10^{4}$ for $T=1.0,0.4$, and 0.2 , respectively. In the previous simulations on nonentangled polymer melts, ${ }^{24,25,28-30,35}$ numerical results were consistent with the Rouse dynamics for small $p$ (large-scale motions), but deviations are enhanced for large $p$ (small-scale motions) in supercooled states. Furthermore, we give the expression for the stress relaxation function in the Rouse model:

$$
G_{R}(t)=\frac{n k_{B} T}{N} \sum_{p=1}^{N-1} \exp \left(-2 t / \tau_{p}\right),
$$

which is equal to $n k_{B} T(N-1) / N$ at $t=0$ and decays as $n k_{B} T N^{-1} \exp \left(-2 t / \tau_{R}\right)$ for $t \gtrsim \tau_{R}$. Since $G(t)$ is much larger than $G_{R}(t)$ in the relatively short time region $t<\tau_{s}$, they can coincide only in the late stage.

Figure 2 shows the van Hove self-correlation function:

$$
F_{q}(t)=\frac{1}{N} \sum_{j=1}^{N}\left\langle\exp \left[i \boldsymbol{q} \cdot \Delta \boldsymbol{R}_{j}(t)\right]\right\rangle,
$$

where $q=2 \pi, \Delta \boldsymbol{R}_{j}(t)=\boldsymbol{R}_{j}\left(t+t_{0}\right)-\boldsymbol{R}_{j}\left(t_{0}\right)$ is the displacement vector of the $j$ th bead in the time interval $\left[t_{0}, t_{0}+t\right]$. The peak wave number of the static structure factor is given by $q \cong 2 \pi$. We define the $\alpha$ relaxation time $\tau_{\alpha}$ from the condition;

$$
F_{q}\left(\tau_{\alpha}\right)=e^{-1} \quad(q=2 \pi) .
$$




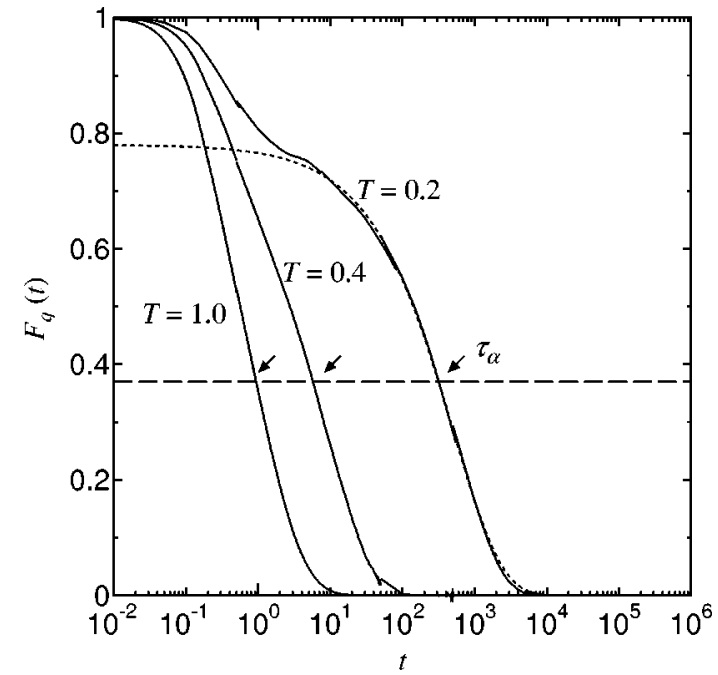

FIG. 2. The van Hove self-correlation function $F_{q}(t)$ at $q=2 \pi$ for $T$ $=1.0,0.4$, and 0.2 on a semilogarithmic scale. The dotted line represents the stretched exponential decay $\propto \exp \left[-\left(t / \tau_{\alpha}\right)^{0.64}\right]$.

As reported in the literature, $\tau_{\alpha}$ increases drastically with lowering $T .{ }^{24-26,28,29}$ In our case, we obtained $\tau_{\alpha}=0.91,5.8$, and 310 for $T=1.0,0.4$, and 0.2 , respectively. At $T=0.2$, where the particle motions are considerably jammed, $F_{q}(t)$ exhibits a two-step relaxation and may be excellently fitted to the stretched exponential decay $\left(\propto \exp \left[-\left(t / \tau_{\alpha}\right)^{0.64}\right]\right)$ for $t$ $\geq 10$. Thus our system at $T=0.2$ has characteristic features of a supercooled state, although its melting temperature is unknown. We find $\tau_{\alpha} \sim 10^{-2} \zeta b^{2} / k_{B} T$ and $\tau_{R} \sim N^{2} \tau_{\alpha}$ at any $T$. In particular, for $T=0.2$, we obtain

$$
\tau_{\alpha} \cong 0.017 \zeta b^{2} / k_{B} T, \quad \tau_{R} \cong 1.9 N^{2} \tau_{\alpha} .
$$

The friction coefficient $\zeta$ in the mapped Rouse model grows strongly as $T$ is lowered in supercooled states.

Now we discuss the linear viscoelastic behavior in supercooled states. In terms of $\Pi_{x y}^{\mathrm{T}}(t)$ in Eq. (2.3), the stress relaxation function $G(t)$ is written as ${ }^{33,34}$

$$
G(t)=\left\langle\Pi_{x y}^{\mathrm{T}}\left(t+t_{0}\right) \Pi_{x y}^{\mathrm{T}}\left(t_{0}\right)\right\rangle / k_{B} T V .
$$

Figure 3 shows the numerical data of $G(t)$ where the average over the initial time $t_{0}$ was taken in one very long run $\left(\sim 100 \tau_{R}\right)$. Here the data become noisy at very large time separation $t \gtrsim \tau_{R}$, where we pick up the correlation decreased down to $10^{-4}-10^{-5}$ of the initial value. In the very early stage $t \leqq 1, G(t)$ oscillates rapidly due to the vibrations of the bond vectors $\boldsymbol{b}_{j}=\boldsymbol{R}_{j}-\boldsymbol{R}_{j+1}$. The initial value $G(0)$ takes a large value $\left(\sim 100\right.$ in units of $\left.\epsilon / \sigma^{3}\right)$ nearly independent of $T$. For $T=0.2, G(t)$ can be nicely fitted to the the stretched exponential form (1.1) with $G_{0} \cong 5, \tau_{s}=90$ $\cong 0.33 \tau_{\alpha}$, and $c=0.5$ in the time region $1 \leqq t \leqq 10 \tau_{s}$. For $t$ $\gtrsim 50 \tau_{s}$ it approaches the Rouse stress relaxation function $G_{R}(t)$ in Eq. (3.5). Here the hierarchical relation $G(0)$ $\gg G_{0} \gg T / N$ is a characteristic feature of glassy polymers, where $T / N$ is the shear modulus of the Rouse model. The zero-frequency Newtonian viscosity is given by $\eta(0)$ $=\int_{0}^{\infty} d t G(t)$, so it consists of the glassy (monomeric) part:

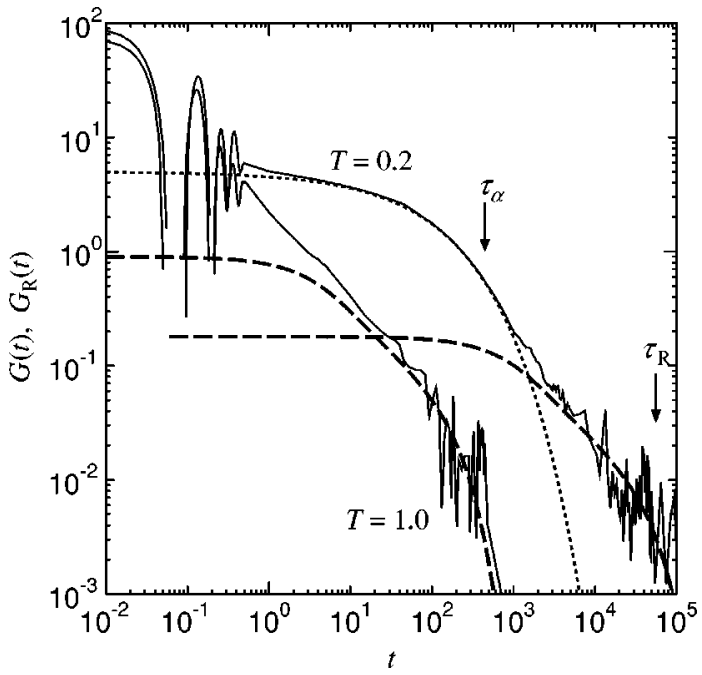

FIG. 3. Stress relaxation function $G(t)$ in Eq. (3.10) (thin-solid lines) at $T$ $=0.2$ in a supercooled state and $T=1$ in a normal liquid state. For $T$ $=0.2$, it can be fitted to the stretched exponential form, $\exp \left[-\left(t / \tau_{s}\right)^{0.5}\right]$ with $\tau_{s}=90$, (dotted line) for $1 \lesssim t \lesssim 10^{3}$ and tends to the Rouse relaxation function $G_{R}(t)$ (bold-dashed lines) at latter times.

$$
\eta_{G}=\int_{0}^{\infty} d t G_{G}(t) \sim 10 \tau_{s}
$$

and the Rouse (polymeric) part:

$$
\eta_{R}=\int_{0}^{\infty} d t G_{R}(t) \cong 0.808 T N^{-1} \tau_{R}
$$

The ratio $\eta_{G} / \eta_{R}$ is of order $1 / T N$. They are of the same order in the present case of $N=10$ and $T=0.2$. However, we should have $\eta_{G} \ll \eta_{R}$ for much larger $N$.

To examine the orientation of the bonds, we consider the orientational tensor:

$$
Q_{\alpha \beta}(t)=\frac{1}{M} \sum_{\text {chain }} \frac{1}{N-1} \sum_{j=1}^{N-1} \frac{b_{j \alpha}}{b_{\min }} \frac{b_{j \beta}}{b_{\min }},
$$

where $b_{\min }$ is defined below Eq. (2.2) and $b_{\min }^{-1} \boldsymbol{b}_{j}$ are the normalized bond vectors since $\left|\boldsymbol{b}_{j}\right| \cong b_{\min }$ as stated below Eq. (2.2). Notice that in the Rouse model the space integral of the entropic stress tensor is given by the expression $\sigma_{\alpha \beta}^{\mathrm{e}}$ $\equiv\left(3 k_{B} T b_{0}^{2} / b^{2}\right) Q_{\alpha \beta}$, where $b$ determined by Eq. (3.3) appears instead of $b_{0}$. To compare our microscopic system and the simplified Rouse model, we calculated the timecorrelation function;

$$
G_{b}(t)=\left\langle\sigma_{x y}^{\mathrm{e}}\left(t+t_{0}\right) \sigma_{x y}^{\mathrm{e}}\left(t_{0}\right)\right\rangle / k_{B} T V,
$$

by integrating Eq. (2.5) in quiescent states. As shown in Fig. $4, G_{b}(t)$ is fairly close to $G_{R}(t)$ in Eq. (3.6) from the Rouse model. In particular, for $t \geqq 0.1 \tau_{R}$, we find $G(t) \cong G_{b}(t)$ $\cong G_{R}(t)$.

\section{STEADY STATE BEHAVIOR IN SHEAR FLOW}

In Fig. 5, we display the steady-state viscosity $\eta(\dot{\gamma})$ $\equiv \sigma_{x y} / \dot{\gamma}$ obtained at $T=0.2,0.4$, and 1 , where the time average of the stress was taken. The crossover shear rate from 


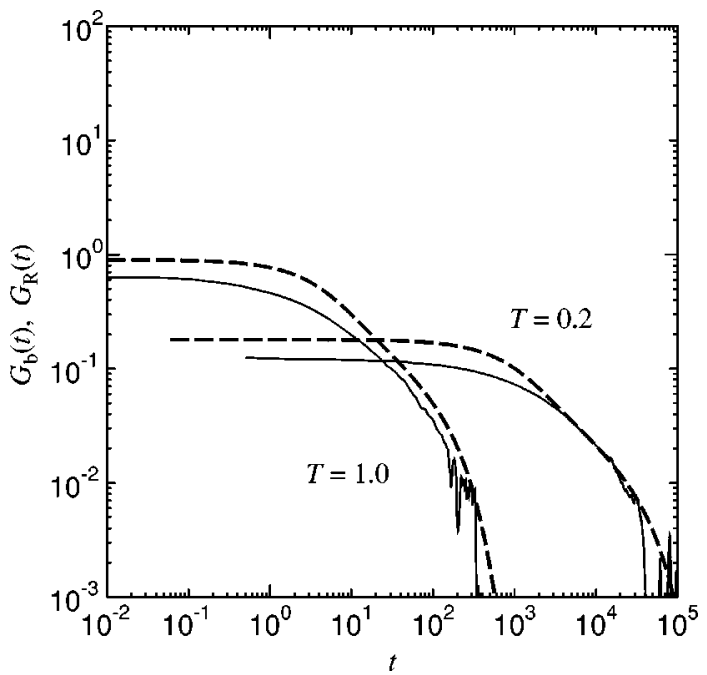

FIG. 4. Comparison of the time-correlation function $G_{b}(t)$ in Eq. (3.14) (thin-solid lines) and the Rouse relaxation function $G_{R}(t)$ in Eq. (3.6) (bolddashed lines). The latter is also shown in Fig. 3.

Newtonian to shear-thinning behavior is given by $\tau_{R}^{-1}$ $\sim N^{-2} \tau_{\alpha}^{-1}$. We may introduce the Weisenberg number $W i$ by

$$
W i=\dot{\gamma} \tau_{R} \sim \dot{\gamma} N^{2} \tau_{\alpha} .
$$

In the non-Newtonian regime, we have $W i>1$. The shear stress at the crossover is of order $n k_{B} T N^{-1}$, which is the elastic modulus of the Rouse model. The horizontal arrows indicate the linear Rouse viscosity $\eta_{R}$ in Eq. (3.12), while the vertical arrows indicate the points at which $\dot{\gamma}=\tau_{R}^{-1}$. In particular, the curve of $T=0.2$ may be fitted to

$$
\eta(\dot{\gamma}) \propto \dot{\gamma}^{-\nu}
$$

with $\nu \cong 0.7$ for $\dot{\gamma} \tau_{R} \gtrsim 1$. The $\eta(\dot{\gamma})$ becomes insensitive to $T$ for very high shear $(W i \gg 1)$. However, in MD simulations of short chain systems in normal liquid states, ${ }^{9-12}$ similar shear thinning has been reported, where the crossover shear is much higher. In MD simulations of supercooled simple

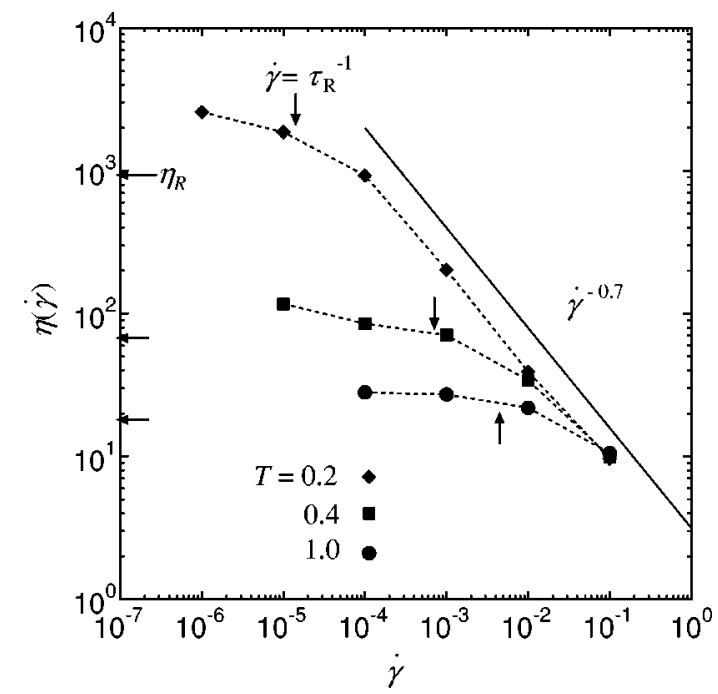

FIG. 5. Steady-state viscosity $\eta(\dot{\gamma})$ vs shear $\dot{\gamma}$ for $T=0.2,0.4$, and 1 . A line of slope -0.7 is a view guide.

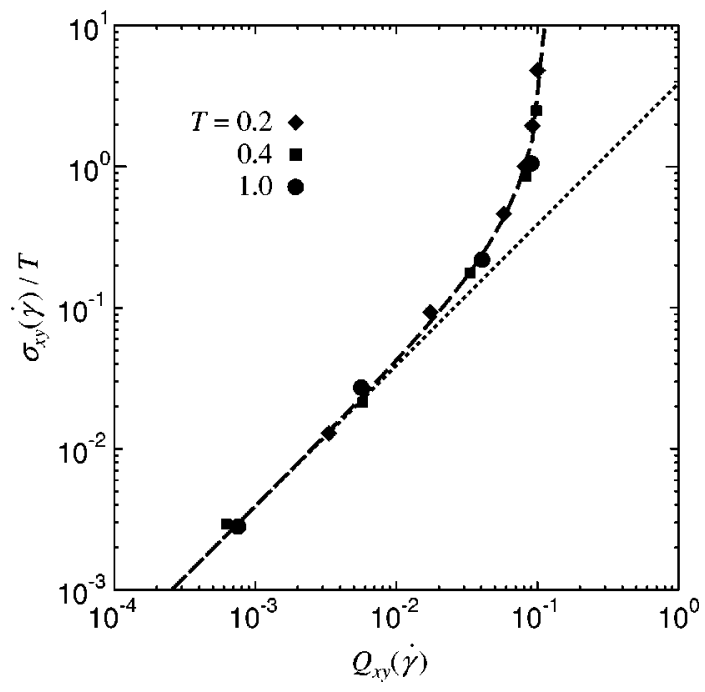

FIG. 6. Universal stress-optical relation $\sigma_{x y} / T$ vs $Q_{x y}$ in steady states under shear flow for $T=0.2,0.4$, and 1 .

binary mixtures, ${ }^{5-7}$ shear-thinning becomes apparent for $\dot{\gamma}$ $\geq \tau_{\alpha}^{-1}$, where $\tau_{\alpha}$ grows at low $T$ and which is consistent with Eq. (4.1) if we set $N=1$.

To demonstrate the stress-optical law in steady states, we show steady-state data of $\sigma_{x y} / T$ vs $Q_{x y}$ for $T=0.2,0.4$, and 1 in Fig. 6. If the electric polarization tensor of a bead is uniaxial along the bond direction, the deviatoric part of the dielectric tensor is proportional to that of the tensor $Q_{\alpha \beta}$ in Eq. (3.13). In accord with the experiment, ${ }^{16}$ our data collapse onto a universal curve independent of $T$ both in the linear $\left(Q_{x y} \lesssim 0.05\right)$ and nonlinear $\left(Q_{x y} \geq 0.05\right)$ regimes.

We next consider anisotropy of chain conformations in shear flow. In Fig. 7(a), we plot the $x-y$ cross section $(z$ $=0$ ) of the steady state bead distribution function:

$$
g_{s}(\boldsymbol{r})=\frac{1}{N} \sum_{j=1}^{N}\left\langle\delta\left(\boldsymbol{R}_{j}-\boldsymbol{R}_{G}-\mathbf{r}\right)\right\rangle
$$

where $\dot{\gamma}=10^{-4}, T=0.2$, and $\boldsymbol{R}_{G}=N^{-1} \sum_{j=1}^{N} \boldsymbol{R}_{j}$ is the center of mass of a chain. In Fig. 7(b), we also plot the structure factor in the $q_{x}-q_{y}$ plane $\left(q_{z}=0\right)$ :

$$
S(\boldsymbol{q})=\frac{1}{N^{2}} \sum_{i, j=1}^{N}\left\langle\exp \left[i \boldsymbol{q} \cdot\left(\boldsymbol{R}_{i}-\boldsymbol{R}_{j}\right]\right\rangle\right.
$$
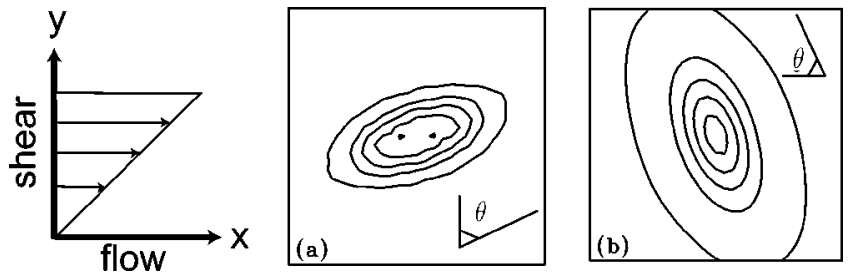

FIG. 7. (a) Isointensity curves of $g_{s}(\mathbf{r})$ in Eq. (4.3) in the $x-y$ plane $(-3.75<x, y<3.75, z=0)$; (b) those of the incoherent structure factor $S(\mathbf{q})$ in Eq. (4.4) in the $q_{x}-q_{y}$ plane $\left(-\pi<q_{x}, q_{y}<\pi, q_{z}=0\right.$ ). The values on the isolines are $0.01+0.02 n$ in (a) and $0.1+0.2 n$ in (b) with $n=0,1,-, 4$ from outer to inner. Here $T=0.2, \dot{\gamma}=10^{-4}$, and the flow is in the horizontal $(x)$ direction. The $\theta$ is the angle between the average chain shapes and the $y$ axis. 


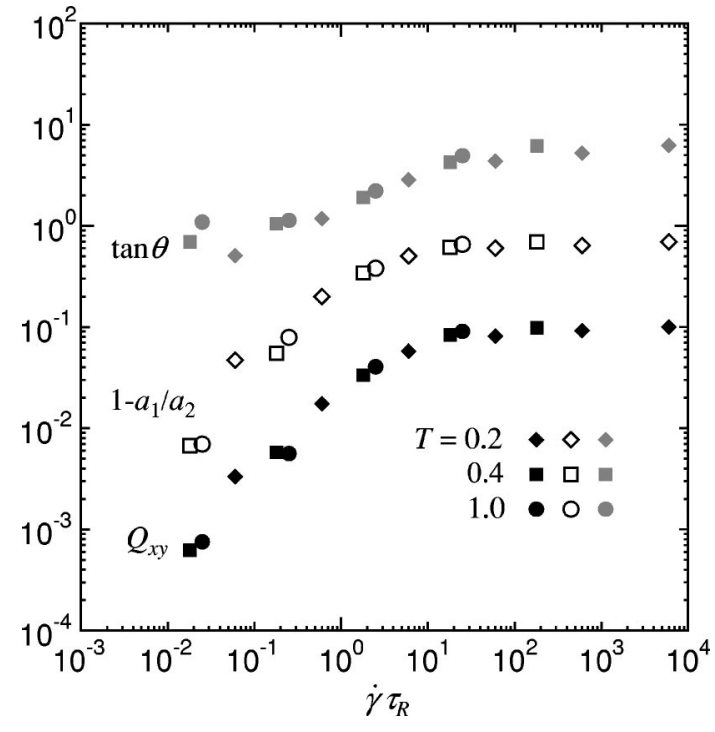

FIG. 8. $\tan \theta, 1-a_{1} / a_{2}$, and $Q_{x y}$ vs $\dot{\gamma} \tau_{R}$ in steady states.

which is proportional to the scattering intensity from labeled chains in shear. ${ }^{37}$ In these figures $\theta$ is the relative angle of the ellipses with respect to the $y$ (shear gradient) direction. These figures demonstrate high elongation of the chains for $\dot{\gamma}>\tau_{R}^{-1}$. As will be shown in Fig. 8 below, they tend to saturate into the shapes shown in Fig. 7 once $\dot{\gamma}$ exceeds $\tau_{R}^{-1}$.

Let us define the tensor,

$$
\begin{aligned}
I_{\alpha \beta} & =\frac{1}{N^{2}} \sum_{i=1}^{N} \sum_{j=1}^{N}\left\langle\left(R_{i \alpha}-R_{j \alpha}\right)\left(R_{i \beta}-R_{j \beta}\right)\right\rangle \\
& =\frac{2}{N} \sum_{j=1}^{N}\left\langle\left(R_{j \alpha}-R_{G \alpha}\right)\left(R_{j \beta}-R_{G \beta}\right)\right\rangle .
\end{aligned}
$$

For small $\boldsymbol{q}=\left(q_{x}, q_{y}, 0\right), S(\boldsymbol{q})$ is expanded as

$$
\begin{aligned}
S(\boldsymbol{q}) & =1-\frac{1}{2} \sum_{\alpha, \beta=x, y} I_{\alpha \beta} q_{\alpha} q_{\beta}+\cdots \\
& =1-\frac{1}{2} a_{1}^{2}\left(\boldsymbol{q} \cdot \boldsymbol{e}_{1}\right)^{2}-\frac{1}{2} a_{2}^{2}\left(\boldsymbol{q} \cdot \boldsymbol{e}_{2}\right)^{2}+\cdots,
\end{aligned}
$$

where $\left\{\boldsymbol{e}_{1}, \boldsymbol{e}_{2}\right\}$ and $\left\{a_{1}^{2}, a_{2}^{2}\right\}$ are the unit eigenvectors and eigenvalues of the tensor $I_{\alpha \beta}(\alpha, \beta \in x, y)$. The two lengths $a_{1}$ and $a_{2}$ correspond to the shorter and longer radii in the principal axes of the ellipses. In terms of $\theta$, we have $\boldsymbol{e}_{1}$ $=(-\sin \theta, \cos \theta)$ and $\boldsymbol{e}_{2}=(\cos \theta, \sin \theta)$ in the $x-y$ plane. In Fig. 8, we display $\tan \theta=-e_{1 y} / e_{1 x}, 1-a_{1} / a_{2}$, and the $x y$ component of the alignment tensor $Q_{x y}$ in Eq. (3.13). All these quantities represent the degree of deformations of chain conformations in shear flow. They are insensitive to $T$ if plotted versus $\dot{\gamma} \tau_{R}$. For $\dot{\gamma} \tau_{R} \lesssim 1, \tan \theta$ is close to $1\left(\theta \cong 45^{\circ}\right)$ and both $1-a_{1} / a_{2}$ and $Q_{x y}$ linearly increase with increasing $\dot{\gamma} \tau_{R}$. For $\dot{\gamma} \tau_{R} \gg 1$, these quantities saturate into limiting values. At $T=0.2$, they are

$$
\theta \cong 80^{\circ}, \quad a_{1} / a_{2} \cong 0.3, \quad Q_{x y} \cong 0.1 .
$$

These are consistent with $Q_{x y} \sim \sin \theta \cos \theta$.

\section{TRANSIENT VISCOELASTIC BEHAVIOR}

In Fig. 9(a), we plot the viscosity growth function $\sigma_{x y}(t) / \dot{\gamma}$ after application of shear $\dot{\gamma}$ at $t=0$ for $T=0.2$, where the solid lines are the averages over ten independent runs. The system was at rest for $t<0$. In the initial stage $\dot{\gamma} t \lesssim 0.1$, it evolves following the linear viscoelastic growth

$$
\frac{1}{\dot{\gamma}} \sigma_{x y}(t) \cong \int_{0}^{t} d t^{\prime} G\left(t^{\prime}\right) .
$$

In the nonlinear regime, $\sigma_{x y}(t) / \dot{\gamma}$ tends to the nonNewtonian viscosity $\eta(\dot{\gamma})$. As a guide, we also display the linear growth function $\int_{0}^{t} d t^{\prime} G_{R}\left(t^{\prime}\right)$ in the Rouse model. In the very early time region $1 \ll t \lesssim \tau_{\alpha}$, the growth $\left(\cong G_{0} t\right)$ is much larger than the Rouse initial growth $\left(\cong k_{B} T t\right)$. We can also see small overshoot behavior of the stress at high shear before approach to the steady state.

In Fig. 9(b), the two growth functions $\sigma_{x y}(t)$ and $20 T Q_{x y}(t)$ are displayed at $\dot{\gamma}=10^{-2}$, where $Q_{x y}$ is defined by Eq. (3.13) and the factor $20 T$ is chosen such that the average values of $Q_{x y}$ and $\sigma_{x y}$ coincide in the steady state. If the noises superimposed on the average curves are neglected, these two quantities nearly coincide for $t \gtrsim 10^{2}$. Here we can see clearly that $\sigma_{x y}(t)$ exhibits two peaks at $t \sim 10$ and $t$ $\sim 200$ for $\dot{\gamma}=10^{-2}$. The first peak is characteristic of glassy liquids and present even in supercooled, simple binary mixtures under high shear $\left(\dot{\gamma} \geq \tau_{\alpha}^{-1}\right){ }^{38}$ The second peak obviously arises from overshoot of chain stretching. More pro-
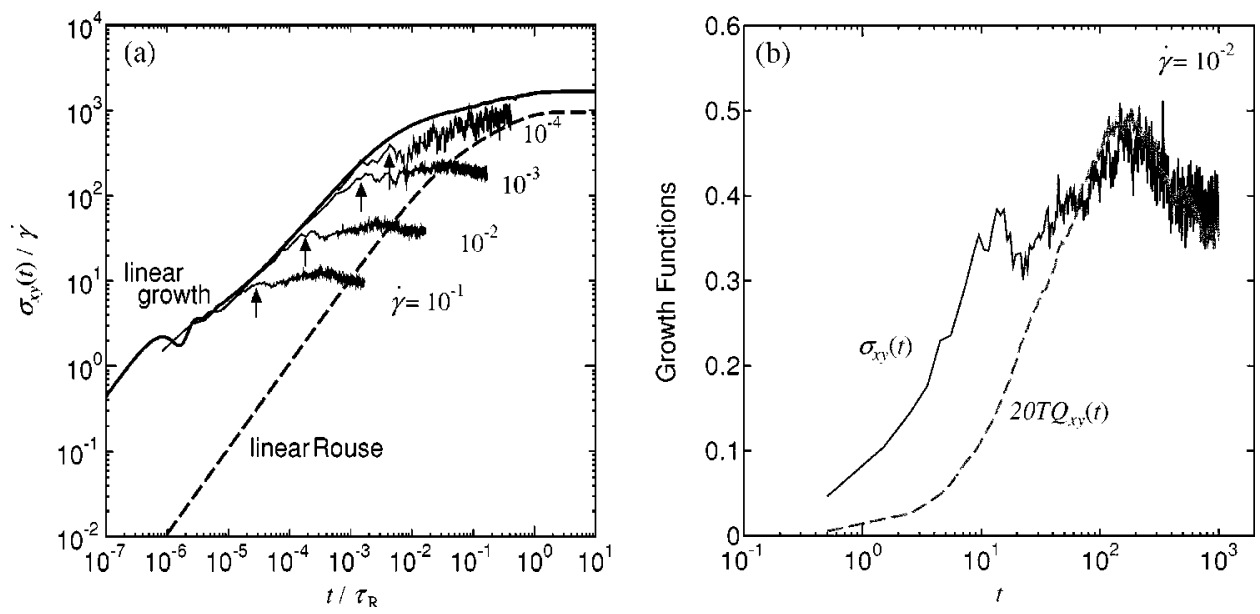

FIG. 9. (a) Shear stress divided by shear rate $\sigma_{x y}(t) / \dot{\gamma}$ vs $t / \tau_{R}$ for $\dot{\gamma}$ $=10^{-1}, 10^{-2}, 10^{-3}, 10^{-4}$, (thin-solid lines) at $T=0.2$ where $\tau_{R}=6 \times 10^{4}$. The curves follow the linear viscosity growth function (bold-solid line) for $\dot{\gamma} t \leqslant 0.1$, but depart from it for $\dot{\gamma} t$ $\gtrsim 0.1$. The linear growth function in the Rouse model is also plotted (bolddashed line). The arrows indicate onset of the nonlinear behavior; (b) $\sigma_{x y}(t)$ and $20 T Q_{x y}(t)$ vs time at $\dot{\gamma}$ $=10^{-2}$ and $T=0.2$. 


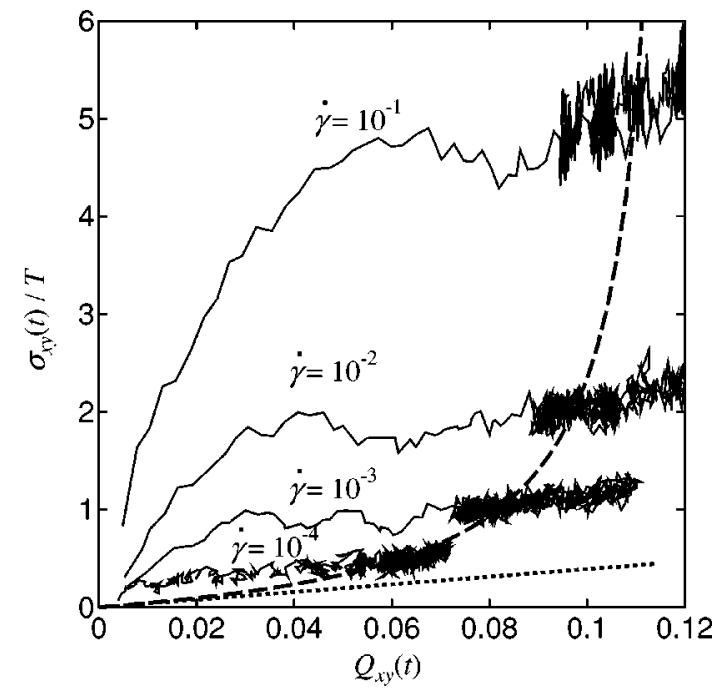

FIG. 10. Parametric plots of $\sigma_{x y}(t) / T$ vs $Q_{x y}(t)$ after application of shear at $t=0$ for $T=0.2$. The curves initially deviate from the universal steady-state curve obtained in Fig. 6 (dashed line) but approach it ultimately. The deviations increase with increasing $\dot{\gamma}$.

nounced overshoot due to chain stretching was already reported at high shear in MD simulation of much longer unentangled alkane chains $\left(\mathrm{C}_{100} \mathrm{H}_{202}\right) .{ }^{12}$ The noise behavior in the curve of $\sigma_{x y}(t)=-\Pi_{x y}^{T} / V$ arises from the thermal fluctuations. The variance of its thermal fluctuations in our finite system is estimated as

$$
\sigma_{\mathrm{fl}}^{2} \equiv\left\langle\left(\sigma_{x y}-\left\langle\sigma_{x y}\right\rangle\right)^{2}\right\rangle \sim G(0) T / V \sim 0.1 T,
$$

where $G(0) \sim 10^{2}$ from Fig. 3. and $V=10^{3}$. In Fig. 9(b), these thermal fluctuations give rise to noisy curves with the fluctuation amplitude being $\sigma_{\mathrm{fl}} / \sqrt{10}$, where 10 is the number of independent runs.

In experiments, ${ }^{16-19}$ the stress-optical relation is transiently violated at low $T$ after application of elongational flow due to the enhancement of the glassy component of the stress. For shear flow, Fig. 10 displays our MD results at $T$ $=0.2$ after application of shear at $t=0$ in a stress-optical diagram, where the solid lines are the averages over ten independent runs. As time goes on, the system traces the curve of a given $\dot{\gamma}$, passes across the dashed curve representing the steady-state universal relation in Fig. 6, and finally returns to the steady-state curve. This crossing behavior arises from simultaneous overshoot in $\sigma_{x y}(t)$ and $Q_{x y}(t)$ as shown in Fig. 9(b). The initial deviation from the steady-state curve becomes larger with increasing $\dot{\gamma}$, as in the experiments of elongational flow. The noises are marked near the steadystate curve, while they are not apparently seen in the initial stage at high shear simply because the density of the data points on the curves is small.

\section{TIME-CORRELATION FUNCTIONS AND TUMBLING IN SHEAR FLOW}

In Fig. 11, we show the end-to-end vector correlation functions $C(t)=\langle\boldsymbol{P}(t) \cdot \boldsymbol{P}(0)\rangle /\left\langle|\boldsymbol{P}|^{2}\right\rangle$ with and without shear flow for various $\dot{\gamma}$ at $T=0.2$. For $\dot{\gamma} \neq 0$, it rapidly decreases, negatively overshoots, and finally approaches zero with

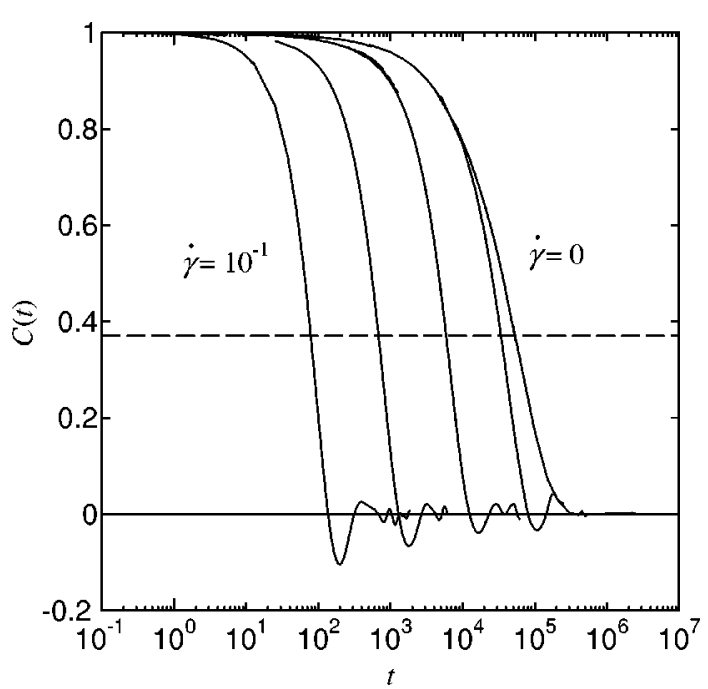

FIG. 11. Normalized time-correlation function of the end-to-end vector $C(t)$ in Eq. (3.4) at $T=0.2$ for $\dot{\gamma}=0,10^{-4}, 10^{-3}, 10^{-2}$, and $10^{-1}$ from right to left on a semilogarithmic scale. The negative overshoot for $\dot{\gamma}>0$ arises from rotational motions of chains.

dumped oscillation superimposed. This oscillatory behavior arises from random rotation of chains in shear flow, which is well known in dilute polymer solutions ${ }^{39-41}$ but has not been reported in polymer melts. This is more evidently seen in Fig. 12, where we show time development of the $x$ component of the end-to-end vector $\boldsymbol{P}_{j}=\boldsymbol{R}_{N}-\boldsymbol{R}_{1}$ of one chain for (a) $\dot{\gamma}=10^{-3}$, (b) $10^{-2}$, and (c) $10^{-1}$ at $T=0.2$. The corresponding Weisenberg number (4.1) is given by $W i$ $=60,600$, and 6000, respectively. In Fig. 12(d), we show chain contours projected onto the $x-y$ plane at points $1-8$ indicated in Fig. 12(c). When the chains change their orientation, their shapes are contracted as in the case of a single chain in solution. ${ }^{40}$ The average period of tumbling is about $35 / \dot{\gamma}$ in our case.

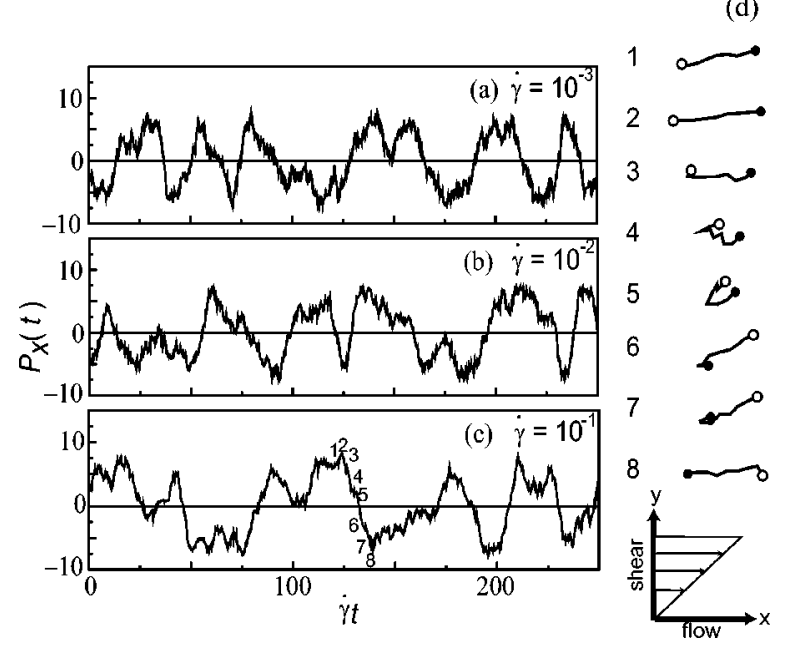

FIG. 12. Time evolution of the $x$ component of the end-to-end vector $P_{x}(t)=X_{N}(t)-X_{1}(t)$ of one chain vs $\dot{\gamma} t$. Here $T=0.2$ and $\dot{\gamma}=10^{-3}$ (a), $10^{-2}(\mathrm{~b})$, and $10^{-1}$ (c) from above. Typical tumbling motions at the points $1-8$ indicated in $(\mathrm{c})$ are shown in $(\mathrm{d})$, where the chain conformations are projected on the $x-y$ plane. 


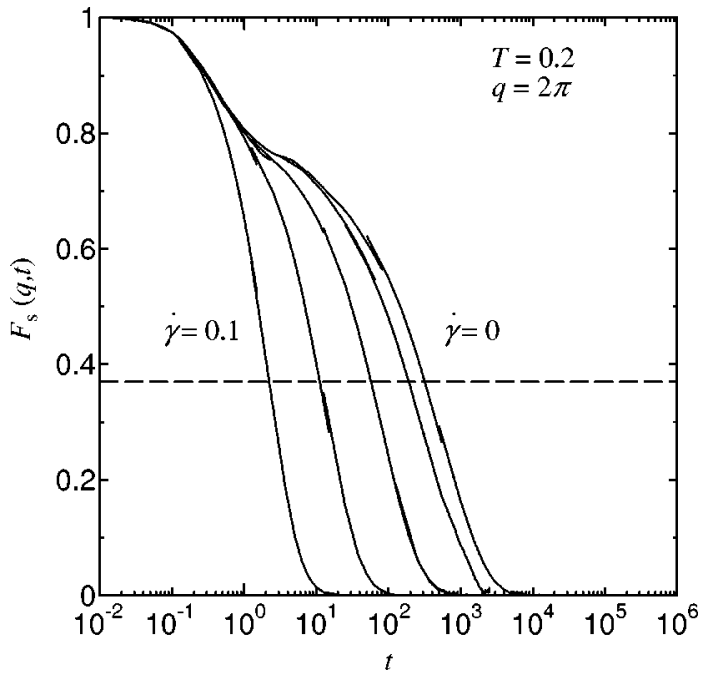

FIG. 13. Van Hove self correlation function Eq. (3.7) with Eq. (6.1) at $T$ $=0.2$ for $\dot{\gamma}=0,10^{-4}, 10^{-3}, 10^{-2}$, and $10^{-1}$ from right to left on a semilogarithmic scale.

We may introduce the van Hove time-correlation function (2.14) even in shear flow if the particle displacement vector is redefined as ${ }^{6}$

$$
\Delta \boldsymbol{R}_{j}(t)=\boldsymbol{R}_{j}\left(t+t_{0}\right)-\boldsymbol{R}_{j}\left(t_{0}\right)-\dot{\gamma} \int_{0}^{t} d t^{\prime} Y_{G}\left(t_{0}+t^{\prime}\right) \boldsymbol{e}_{x},
$$

where $Y_{G}$ is the $y$ component of $\boldsymbol{R}_{G}=N^{-1} \sum_{j=1}^{N} \boldsymbol{R}_{j}$. From the net displacement, the first two terms, we have subtracted the flow-induced displacement (the last term). Figure 13 shows $F_{q}(t)$ with $q=2 \pi$ for various $\dot{\gamma}$ at $T=0.2$. Comparison of this figure with Fig. 2 suggests that applying shear is analogous to raising the temperature. This tendency was already reported for the case of supercooled binary mixtures. ${ }^{6,42}$

We introduce the shear-dependent Rouse time $\tau_{R}$ $=\tau_{R}(T, \dot{\gamma})$ and the $\alpha$ relaxation time $\tau_{\alpha}=\tau_{\alpha}(T, \dot{\gamma})$ by

$$
C\left(\tau_{R}\right)=e^{-1}, \quad F_{q}\left(\tau_{\alpha}\right)=e^{-1} .
$$

We may then examine how shear can accelerate the motions of chains and individual beads in shear flow. Figure 14 shows $\tau_{R}$ and $\tau_{\alpha}$ as functions of $\dot{\gamma}$ at $T=0.2$. In our short chain system, both $\tau_{R}$ and $\tau_{\alpha}$ decrease for $\dot{\gamma} \geq \tau_{R}(T, 0)^{-1}$. Our data at $T=0.2$ are consistent with

$$
\begin{aligned}
& \tau_{R}(T, \dot{\gamma})^{-1}=\tau_{R}(T, 0)^{-1}\left[1+A_{R} \dot{\gamma}\right], \\
& \tau_{\alpha}(T, \dot{\gamma})^{-1}=\tau_{\alpha}(T, 0)^{-1}\left[1+\left(A_{\alpha} \dot{\gamma}\right)^{\mu}\right],
\end{aligned}
$$

where $A_{R} \cong 10^{4} \sim \tau_{R}(T, 0), A_{\alpha} \cong 6000 \simeq 20 \tau_{\alpha}(T, 0)$, and $\mu$ $\cong 0.77$. The average tumbling period in Fig. 12 is about $4 \tau_{R}(T, \dot{\gamma})$. For simple supercooled liquids we already introduced the van Hove time-correlation function in shear flow ${ }^{6}$ and obtained $\tau_{\alpha}(T, \dot{\gamma})^{-1}=\tau_{\alpha}(T, 0)^{-1}\left[1+A_{\alpha} \dot{\gamma}\right]$ with $A_{\alpha}$ $\sim \tau_{\alpha}(T, 0)$.

The sensitive shear dependence of $\tau_{\alpha}(T, \dot{\gamma})$ predicted by Eq. (6.4) suggests potential importance of dielectric measurements in shear flow. ${ }^{6}$ As a first experiment, Matsuyama et al. measured the dielectric loss function $\epsilon^{\prime \prime}(\omega, \dot{\gamma})$ in steady shear $\dot{\gamma}$ in oligostyrene and polyisoprene melts. ${ }^{19}$ In the

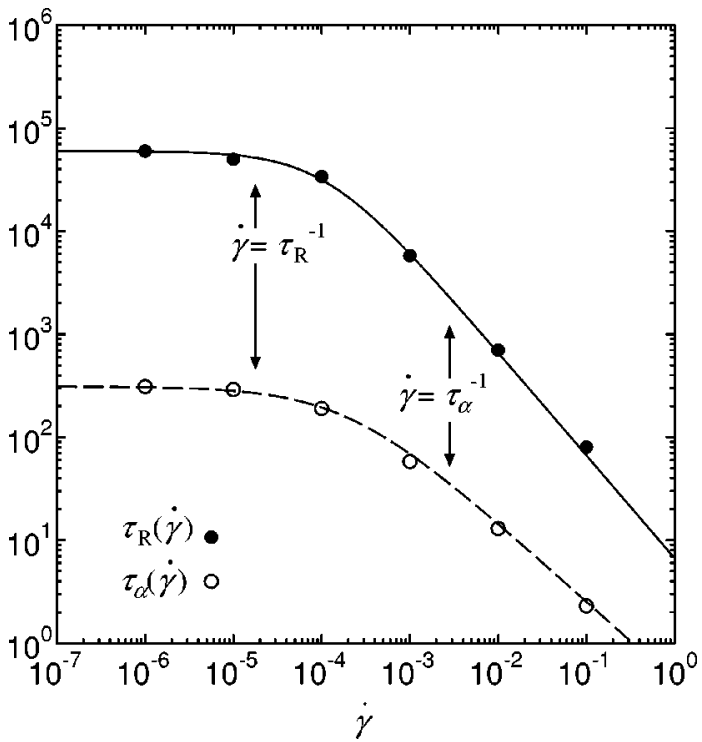

FIG. 14. Two relaxation times $\tau_{R}(\dot{\gamma})$ and $\tau_{\alpha}(\dot{\gamma})$ as functions of shear $\dot{\gamma}$ at $T=0.2$ determined from Eq. (6.2). Both these times decrease for $\dot{\gamma}$ $\gtrsim \tau_{R}(0)^{-1} \sim N^{-2} \tau_{\alpha}(0)^{-1}$ in our short chain system. The solid and dashed lines represent Eqs. (6.3) and (6.4), respectively. The slopes of the curves at high shear are -1 for $\tau_{R}(\dot{\gamma})$ and -0.77 for $\tau_{\alpha}(\dot{\gamma})$.

former melt at $T=42^{\circ} \mathrm{C}, \epsilon^{\prime \prime}(\omega, \dot{\gamma})$ decreased nonlinearly as a function of $\dot{\gamma}$ at low frequencies $\left(\omega \leqq 10^{5} \mathrm{~s}^{-1}\right)$ in the shear-thinning regime $\left(\dot{\gamma} \geqslant 15 \mathrm{~s}^{-1}\right)$. Their finding indicates that $\tau_{\alpha}$ decreases as a function of $\dot{\gamma}$ in the non-Newtonian regime, consistently with Eq. (6.4), More systematic dielectric measurements in supercooled systems under shear flow are very informative.

\section{SUMMARY}

We have performed very long MD simulations of a supercooled polymer melt composed of $M=100$ short chains with bead number $N=10$ in quiescent and sheared conditions. Here we summarize our main simulation results together with remarks.

(i) The stress relaxation function $G(t)$ is shown to follow a stretched exponential decay (1.1) on the scale of the $\alpha$ relaxation time $\tau_{\alpha}$ and then the Rouse relaxation (3.6) on the scale of $\tau_{R}$.

(ii) The nonlinear shear regime sets in at extremely small shear rate of order $\tau_{R}^{-1}$ in supercooled states, where marked shear thinning and shape changes of chains are found. Scattering and birefringence experiments from weakly sheared melts near $T_{g}$ seem to be very promising.

(iii) In the nonlinear shear regime, each chain undergoes random tumbling in our melt as in the case of isolated polymer chains in shear flow. It is of great interest how this effect is universal in solutions and melts and how it influences macroscopic rheological properties. For example, we are interested in whether or not such tumbling occurs in sheared entangled polymers.

(iv) Transient stress divided by $\dot{\gamma}$ after application of shear flow obeys the linear growth $\int_{0}^{t} d t^{\prime} G\left(t^{\prime}\right) \simeq G_{0} t$ for strain less than 0.1 and then saturates into a non-Newtonian steady-state viscosity. This initial growth is much steeper 
than that predicted by the Rouse model. As a result, the stress-optical relation does not hold transiently under deformation even in the linear (zero-shear) limit. Its violation is more enhanced for larger shear rates. These are consistent with the experiments.

(v) The time-correlation functions in shear flow are calculated for the end-to-end vector and the modified particle displacement in Eq. (6.1). The former represents the relaxation of chain conformations, while the latter the monomeric relaxation on the spatial scale of the particle distance. We can then determine the shear-dependent relaxation times, $\tau_{R}(T, \dot{\gamma})$ and $\tau_{\alpha}(T, \dot{\gamma})$. They decrease nonlinearly and behave differently as functions of $\dot{\gamma}$ in the nonlinear shear regime as in Eqs. (6.3) and (6.4). It is then of great interest how these times behave in strong shear for much larger $N$. We conjecture that if $N$ is sufficiently large, shear should first influence the overall chain conformations, while it does not much affect the monomeric relaxations. We propose dielectric measurements in shear flow, which should give information of $\tau_{\alpha}(T, \dot{\gamma}) .{ }^{19}$ In addition, as demonstrated in Fig. 13, the effect of shear on the van Hove self-correlation function is analogous to raising the temperature above $T_{g}$ as in supercooled binary mixtures. 6,42

\section{ACKNOWLEDGMENTS}

The authors would like to thank T. Inoue for valuable discussions on the stress-optical relation. This work is supported by Grants in Aid for Scientific Research from the Ministry of Education, Science, Sports and Culture of Japan. Computations have been performed at the Human Genome Center, Institute of Medical Science, University of Tokyo.

${ }^{1}$ S. Matsuoka, Relaxation Phenomena in Polymers (Oxford, New York, 1992).

${ }^{2}$ G. R. Stroble, The Physics of Polymers (Springer, Heidelberg, 1996).

${ }^{3}$ M. Doi and S. F. Edwards, The Theory of Polymer Dynamics (Clarendon, Oxford, 1986).

${ }^{4}$ J. H. Simmons, R. Ochoa, K. D. Simmons, and J. J. Mills, J. Non-Cryst. Solids 105, 313 (1988).

${ }^{5}$ R. Yamamoto and A. Onuki, Europhys. Lett. 40, 61 (1997).

${ }^{6}$ R. Yamamoto and A. Onuki, Phys. Rev. E 58, 3515 (1998).

${ }^{7}$ R. Yamamoto and A. Onuki, J. Phys.: Condens. Matter 29, 6323 (2000).

${ }^{8}$ M. Kröger, W. Loose, and S. Hess, J. Rheol. 37, 1057 (1993).

${ }^{9}$ S. Chynoweth and Y. Michopoulos, Mol. Phys. 81, 133 (1994).

${ }^{10}$ R. Khare and J. de Pablo, J. Chem. Phys. 107, 6956 (1997).
${ }^{11}$ J. D. Moore, S. T. Cui, H. D. Cochran, and P. T. Cummings, J. NonNewtonian Fluid Mech. 93, 83 (2000); 93, 101 (2000).

${ }^{12}$ S. Bair, C. McCabe, and P. T. Cummings, Phys. Rev. Lett. 88, 058302 (2002).

${ }^{13}$ P. H. Mott, A. S. Aragon, and U. W. Suter, Philos. Mag. A 67, 931 (1993).

${ }^{14}$ A. S. Aragon, V. V. Bulatov, P. H. Mott, and U. W. Suter, J. Rheol. 39, 377 (1995).

${ }^{15}$ A. Onuki and M. Doi, J. Chem. Phys. 85, 1190 (1986).

${ }^{16}$ R. Muller and J. J. Pesce, Polymer 35, 734 (1994).

${ }^{17}$ M. Kröger, C. Luap, and R. Muller, Macromolecules 30, 526 (1997).

${ }^{18}$ T. Inoue, D. S. Ryu, and K. Osaki, Macromolecules 31, 6977 (1998).

${ }^{19}$ M. Matsuyama, H. Watanabe, T. Inoue, and K. Osaki, Macromolecules 31, 7973 (1998).

${ }^{20}$ K. Kremer and G. S. Grest, J. Chem. Phys. 92, 5057 (1990).

${ }^{21}$ I. Carmesin and K. Kremer, Macromolecules 21, 2819 (1988)

${ }^{22}$ H. P. Deutsch and K. Binder, J. Chem. Phys. 94, 2294 (1991).

${ }^{23}$ D. Rigby and R.-J. Roe, J. Chem. Phys. 87, 7285 (1987); R.-J. Roe, J. Chem. Phys. 100, 1610 (1994).

${ }^{24}$ C. Bennemann, W. Paul, K. Binder, and B. Dünweg, Phys. Rev. E 57, 843 (1998); C. Bennemann, J. Baschnagel, and W. Paul, Eur. Phys. J. B 10, 323 (1999); C. Bennemann, J. Baschnagel, W. Paul, and K. Binder, Comput. Theor. Polym. Sci. 9, 217 (1999).

${ }^{25}$ J. Baschnagel, C. Bennemann, W. Paul, and K. Binder, J. Phys.: Condens. Matter 12, 6365 (2000).

${ }^{26}$ N. E. Moe and M. D. Ediger, Phys. Rev. E 59, 623 (1999).

${ }^{27}$ A. van Zon and S. W. de Leeuw, Phys. Rev. E 60, 6942 (1999).

${ }^{28} \mathrm{~K}$. Okun, M. Wolfgardt, J. Baschnagel, and K. Binder, Macromolecules 30, 3075 (1997)

${ }^{29}$ W. Paul and J. Baschnagel, in Monte Carlo and Molecular Dynamics Simulations in Polymer Science, edited by K. Binder (Oxford University Press, New York, 1995), pp. 307-355.

${ }^{30} \mathrm{~K}$. Binder, C. Bennemann, J. Baschnagel, and W. Paul, in Anomalous Diffusion, edited by R. Kutner, A. Pekalski, and K. Sznajd-Weron (Springer, Berlin, 1999), pp. 124-139; K. Binder, J. Baschnagel, C. Bennemann, and W. Paul, J. Phys.: Condens. Matter 11, A47 (1999).

${ }^{31}$ Y. Rouault and K. Kremer, J. Chem. Phys. 111, 3288 (1999).

${ }^{32}$ A. Onuki, Phase Transition Dynamics (Cambridge University Press, Cambridge, 2002).

${ }^{33}$ M. P. Allen and D. J. Tildesley, Computer Simulation of Liquids (Clarendon, Oxford, 1987).

${ }^{34}$ D. J. Evans and G. P. Morriss, Statistical Mechanics of Nonequilibrium Liquids (Academic, New York, 1990).

${ }^{35}$ A. Kopf, B. Dünweg, and W. Paul, J. Chem. Phys. 107, 6945 (1997).

${ }^{36}$ P. H. Verdier, J. Chem. Phys. 45, 2118 (1966).

${ }^{37}$ R. Muller, J. J. Pesce, and C. Picot, Macromolecules 26, 4356 (1993).

${ }^{38} \mathrm{R}$. Yamamoto and A. Onuki (unpublished).

${ }^{39}$ I. Y. Z. Zia, R. G. Cox, and S. G. Mason, Proc. R. Soc. London, Ser. A 300, 421 (1967).

${ }^{40}$ D. E. Smith, H. P. Babcock, and S. Chu, Science 283, 1724 (1999).

${ }^{41}$ P. LeDuc, C. Haber, G. Bao, and D. Wirtz, Nature (London) 399, 564 (1999).

${ }^{42}$ L. Berthier and J.-L. Barrat, Phys. Rev. E 63, 012503 (2001); J. Chem. Phys. 116, 6228 (2002). 